\title{
Erratum to: Endoscopic endonasal trans-sphenoidal approach for pituitary adenomas: Is one nostril enough?
}

\author{
Sheng Han • Xiaoxu Ding • Xinxin Tie • Yuan Liu • \\ Junze Xia • Aihui Yan • Anhua Wu
}

Published online: 24 September 2013

(C) Springer-Verlag Wien 2013

Erratum to: Acta Neurochir (2013) 155:1601-1609

DOI: 10.1007/s00701-013-1788-8

The original version of this article unfortunately contained mistakes. On page 1607 and 1608, the figure legends for Fig 5 and 6 should be interchanged. Figure 5 shows images obtained from a 64-year-old female with a nonfunctioning macroadenoma who underwent the two-nostril approach. Figure 6 comprises images obtained from a 51-year-old man with a GH-secreting macroadenoma who underwent the one-nostril approach.

The online version of the original article can be found at http://dx.doi.org/ 10.1007/s00701-013-1788-8.

S. Han $\cdot$ X. Tie $\cdot$ Y. Liu $\cdot$ J. Xia $\cdot$ A. Wu $(\bowtie)$

Department of Neurosurgery, The First Hospital of China,

Medical University, Nanjing Street 155, Heping District,

Shenyang 110001, China

e-mail: cmuwuanhua@yahoo.com.cn

X. Ding $\cdot$ A. Yan

Department of Otorhinolaryngology, The First Hospital of China,

Medical University, Shenyang, China 